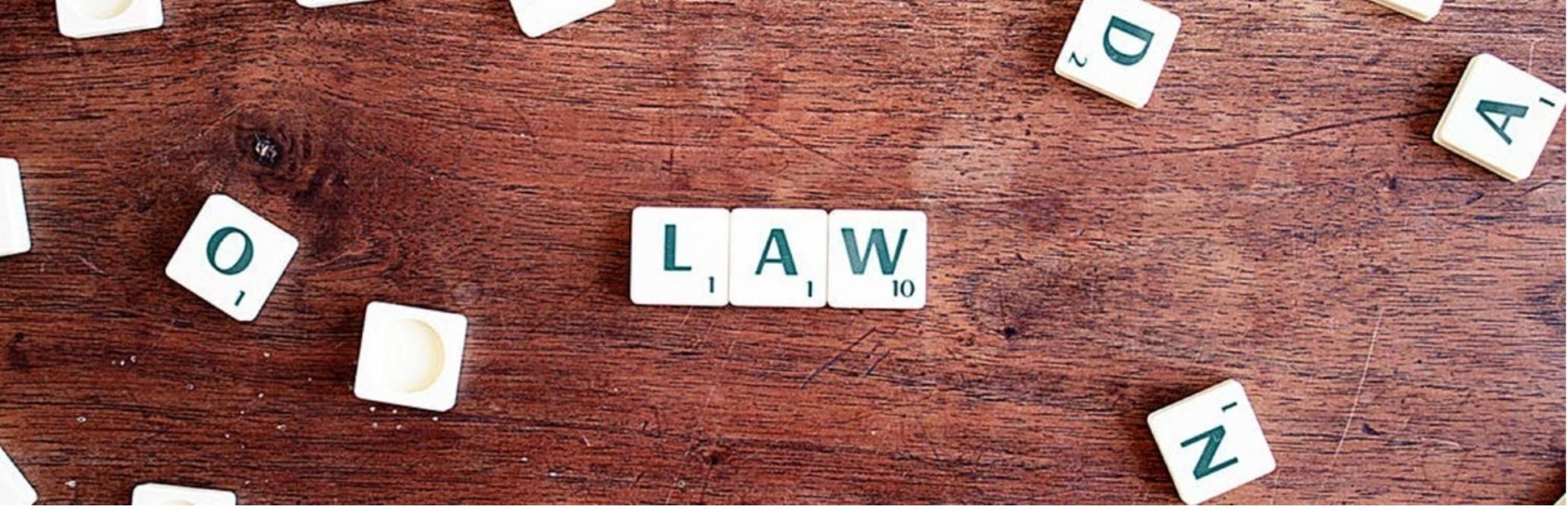

\title{
Quality and Protection of Confidence - Qualität und Vertrauensschutz
}

\author{
Authors: $\quad$ Sigrun Löwisch \\ Submitted: $\quad$ 28. October 2019 \\ Published: $\quad$ 28. October 2019 \\ Volume: 6 \\ Issue: $\quad 10$ \\ Languages: German \\ Keywords: $\quad$ CDU Partei, Law, politics, Psychotherapy law. \\ Categories: Humanities, Social Sciences and Law \\ DOl: $\quad$ 10.17160/josha.6.10.608
}

\section{Abstract:}

For patients and psychotherapists, the passing of the Psychotherapists Act by the Bundestag and the Busdesrat is good news. The understanding between the coalition and the opposition in this matter, which has been controversial for almost 20 years, is also proof of the ability of Germany's much criticized politicians to act. It shows that it is also possible to drill thick boards if the Bundesrat enters into a factoriented discussion and the Länderkammer is not misused as a pure blockade instrument. Sigrün löwisch is a German politician of the CDU. She was a member of the German Bundestag from 1991 to 1998.

\section{JOSHA Jumnalossimene, Humanities and Arts}




\title{
Qualität und Vertrauensschutz
}

\author{
Sigrun Löwisch,
}

Ehemaliger Mitglied des Bundestages und des Gesundheitsausschusses, CDU.

Für Patienten und Psychotherapeuten ist die Verabschiedung des Psychotherapeutengesetzes durch Bundestag und Busdesrat eine gute Nachricht. Die Verständigung von Koalition und Opposition in dieser seit bald 20 Jahren umstrittenen Materie ist aber auch ein Beweis für die Handlungsfähigkeit der vielgescholtenen deutschen Politik. Sie zeigt, dass auch das Bohren sicker Bretter möglich ist, wenn der Bundesrat in eine Sachorientierte Diskussion eintritt, und die Länderkammer nicht als reines Blockadeninstrument missbraucht wird.

Die CDU/CSU hat bei den Beratungen zum Psychotherapeutengesetz drei Ziele verfolgt:

1. Die Gewährleistung einer qualitativ hochstehenden Psychotherapie in den Interessen der Patienten.

2. Die Begründung des Erstzugangsrechts zum Psychologischen Psychotherapeuten für die Versicherten der Gesetzlichen Krankenversicherung (GKV), und damit verbunden die gleichberechtigte Integration der Psychologischen Psychotherapeuten in das System der GKV.

3. Die Stärkung der Eigenverantwortung der Patienten auch im Bereich der Psychotherapie.

Das Psychotherapeutengesetz trägt diesen drei Maximen Rechnung. Es schafft die Grundlagen für eine hochwertige psychotherapeutischen Versorgung in Deutschland. Dem dienen die Qualitätsstandards, die das Psychotherapeutengesetz setzt, ebenso wie der Schutz der Berufzeichnung. Die Bezeichnung „Psychotherapeut“ bleibt künftig den Psycho logischen Psychotherapeuten und den Kinder- und Jugendlichenpsychotherapeuten vorbehalten. Zugleich 
ist es gelungen, Übergangsregelungen zu finden, die allen Behandlern, die schon bisher in versorgungsrelevantem Umfang tätig waren, die Möglichkeit der Approbation und der bedarfsunabhängigen Zulassung zur Versorgung der GKV- Versicherten eröffnen. Der Vertrauensschutz ist garantiert, ohne dass in der Qualität Abstriche gemacht würden. Ein besonderes Augenmerk verdient dabei die sogenannte Erziehungsklausel, nach der sich für Therapeuten, deren berufliches Fortkommen sich wegen der Erziehung eines Kindes verzögert hat, die Übergangsfristen um bis zu drei Jahren verschieben könnten.

Das Psychotherapeutengesetz überwindet das Delegationsverfahren. Psychotherapeuten sind künftig nicht mehr Hilfspersonen eines Artes. Die Patienten haben unmittelbaren Zugang zu ihrem Psychotherapeuten, unabhängig davon, ob dieser eine medizinische, psychologische oder pädagogische Vorbildung hat. Diese Aufwertung findet ihren Niederschlag auch in der sozialrechtlichen Stellung der Psychotherapeuten. Sie werden in die Kassenärztlichen Vereinigungen integriert. Für Psychotherapeuten und psychotherapeutisch tätige Ärzte wird es eine gemeinsame Bedarfsplanung und die gleiche Leistungsvergütung geben. Dabei ist für 1999, wenn das Psychotherapeutengesetz in Kraft tritt, ein Finanzvolumen mit versorgungssicherndem Niveau festgelegt.

Einen wichtigen Punkt stellt die paritätische Besetzung der Leistungserbringerbank des Bundesausschusses der Ärzte und Krankenkassen mit Psychotherapeuten und psychotherapeutisch tätigen Ärzten dar, wenn über die Psychotherapie- Richtlinien beschlossen wird. Die Richtlinien-Therapien sind die Bezugspunkte der im Psychotherapeutengesetz verankerte Qualitätsstandards. Es hat hierzu viele Missverständnisse gegeben. Auch wird unter dem Schlagwort einer Einschränkung der Methodenvielfalt immer wieder gegen die Richtlinienverfahren polemisiert. Die Maßgeblichkeit dieser Verfahren folgt aus der Systematik unserer GKV. Danach werden von der Solidargemeinschaft nur die Kosten solcher Behandlungsverfahren übernommen, die eine ausreichende, zweckmäßige und wirtschaftliche Versorgung der Versicherten gewährleisten ( 91 SGB V). Es ist zwingend geboten, dass die entsprechenden Entscheidungen auf der Fachebene und nicht von Politikern getroffen werden. Nur auf diese Weise ist eine offene und wissenschaftliche Entwicklung der Psychotherapie zu erreichen. Im Schatten der Kostenerstattungspraxis hat die Entwicklung der Richtlinien häufig genug nicht die erforderliche Beachtung gefunden. Dies muss und wird sich ändern. Die 
Mitgestaltung der Diskussion im Bundesausschuss wird sich ein entscheidendes Feld künftiger Verbandsarbeit sein.

Es ist schließlich gelungen, den Aspekt der Eigenverantwortung der Versicherten in das Gesetzgebungsverfahren einzubringen. Die Versicherten werden eine Selbstbeteiligung von 10 DM pro Sitzung leisten. Zuzahlungsfrei bleiben die ersten zwei probatorischen Sitzungen. Die Selbstbeteiligung ist durch eine Überforderungsklausel sozial abgefedert. Übersteigen die Zuzahlungen 2\% der jährlichen Bruttoeinnahmen eines Versicherten, werden sie von der Krankenkasse übernommen. Bei chronisch Kranken beträgt die Belastungsgrenze 1\%. Darüber hinaus ist die e

Eigenbeteiligung in Härtefällen sowie bei Kindern und Jugendlichen unter 18 Jahren ausgeschlossen. Rund 20 Millionen Versicherte bleiben damit von der Zuzahlungspflicht völlig freigestellt. Allen idiologischen Unkenrufen zum Trotz steht damit fest. Das Psychotherapeutengesetz gewährleistet auf soziale Weise den Zugang zu hochqualifizierten Behandlern. 